\title{
PASTOREO ROTADO EN BRACHIARIA BRIZANTHA CV. MARANDU PARA MEJORAR LA PRODUCCIÓN DE LECHE
}

\section{ARTÍCULO ORIGINAL}

SOARES, Marcio Groto ${ }^{1}$, CAMARGO, Simone Cristina², FRASSETTO, Matheus Orlandin ${ }^{3}, \mathrm{ABREU}$, Hélio Mar de ${ }^{4}$

SOARES, Marcio Groto. Et al. Pastoreo rotado en Brachiaria brizantha cv. marandu para mejorar la producción de leche. Revista Científica Multidisciplinar Núcleo do Conhecimento. Año. 06, Ed. 09, Vol. 07, págs. 104-118. Septiembre 2021. ISSN: 2448-0959, Enlace de acceso: https://www.nucleodoconhecimento.com.br/veterinaria-es/pastoreo-rotado, $\quad$ DOI: 10.32749/nucleodoconhecimento.com.br/veterinaria-es/pastoreo-rotado

\section{RESUMEN}

En las propiedades lácteas, para que haya un aumento de la ganancia, es necesario aumentar la eficiencia de los factores de producción, especialmente la nutrición, lo que impacta en el costo fijo. Cada vez más, se busca un equilibrio entre el suministro de piensos y pastos. El método de pastoreo rotacional ha sido adoptado por varios productores, ya que tiene una buena productividad en un área más pequeña. Sin embargo, los pastos utilizados en este tipo de pastoreo, a pesar de tener una excelente calidad, tienen mayores requerimientos nutricionales. El objetivo de este trabajo fue realizar un análisis económico del costo de producción de leche, mediante la implantación de pastoreo rotacional, e intercambio de pastos nativos por Brachiaria brizantha $\mathrm{cv}$. marandu, en una granja familiar. Este trabajo fue elaborado en la ciudad de Foz do Iguaçu. Se utilizó un área de $6300 \mathrm{~m}^{2}$, dividida en 28 potreros

\footnotetext{
${ }^{1}$ Graduado universitario.

2 Doctorado.

${ }^{3}$ Maestro.

${ }^{4}$ Graduado universitario.
}

RC: 99520

Disponible: https://www.nucleodoconhecimento.com.br/veterinaria-es/pastoreorotado 
de $225 \mathrm{~m}^{2}$ mediante cerca eléctrica. El costo total de producción fue de $R \$ 6.047,40$, con un margen positivo de $\mathrm{R} \$ 5.143,61$ por año. De acuerdo con los resultados obtenidos en los análisis económicos, se concluye que la implantación del pastoreo rotacional y el intercambio de pasto nativo por Brachiaria brizantha cv. Marandu es una gestión viable, ya que los ingresos generados por la venta de leche fueron suficientes para cubrir los costos totales de producción.

Palabras clave: Costo, Leche, Forraje, Piquete, Manipulación.

\section{INTRODUCCIÓN}

Brasil ocupa el cuarto lugar en el ranking mundial de producción de leche. En 2018, la región Sur representó el 34\% de la producción total del país (ANUARIO DE LA LECHE, 2019). Solo el estado de Paraná produjo este año un promedio de 4.370 millones de litros de leche, de los cuales el oeste de Paraná fue responsable del 19\% (IBGE, 2018).

El ganado lechero en el estado de Paraná es una actividad típica de la agricultura familiar, representando aproximadamente el $85,1 \%$ de los productores del estado (SEAB, 2018). La mayoría de estos productores utilizan los pastos como la principal fuente de alimento para los animales. El área promedio ocupada con pastizales en el estado es de 16.6 hectáreas (IPARDES, 2009).

Aunque Brasil se destaca en la producción de leche, su productividad es de alrededor de $1.689 \mathrm{~L} / \mathrm{vaca} / \mathrm{año}$, que es inferior a la de Estados Unidos, China, Rusia, Argentina, la Unión Europea y Nueva Zelanda, que producen más de 3 L/vaca/año. Uno de los factores responsables de la baja producción de leche es el uso de pastos poco productivos de baja calidad nutricional, junto con un manejo inadecuado tanto de animales como de pastos (GONÇALVES et al., 2003).

Una forma de intensificar la producción animal en los sistemas de pastoreo es la adopción de forrajes mejorados, más adaptados al clima, con mayor potencial de

RC: 99520

Disponible: https://www.nucleodoconhecimento.com.br/veterinaria-es/pastoreorotado 
producción y mejor calidad (JANK, 2017). Entre los diversos forrajes existentes en Brasil, se destaca la variedad híbrida de la hierba Brachiaria brizantha cv. marandu, por presentar un buen valor nutricional y alta producción de biomasa, adaptándose bien a suelos de fertilidad media y buena, siendo tolerante a la acidez del suelo (FONSECA et al., 2010).

El correcto manejo de los pastos debe ser otro punto a observar en las propiedades del ganado lechero. Uno de los pastos que se ha demostrado viable es el rotacional, donde hay subdivisión del pasto en tres o más piquetes. Este tipo de pastoreo tiene varias ventajas, tales como: mayor uniformidad de pastoreo, mayor uso de forrajes, mayor tasa de siembra y mayor longevidad de las malezas que forman grupos (FUKUMOTO et al., 2010).

Diferentes estudios han mostrado buenos resultados para el ganado lechero, con la implantación del pastoreo rotacional en el pasto Brachiaria brizantha cv. marandu (GONÇALVES, 2003); (DEMSKI, 2013), (MOURA, 2017). Según (ANDRADE, 2008), este tipo de pasto no tolera el pastoreo continuo.

Con la correcta elección del forraje, un manejo adecuado y un mejor aprovechamiento del área de propiedad, se busca generar ahorros al productor, ya que reducirá el costo de producción. Según (GERON, 2012), el coste de producción es la suma de todas las cantidades gastadas en operaciones y servicios utilizados en el proceso productivo de una determinada actividad. Con el estudio de los costes, es posible verificar cómo se están remunerando los recursos utilizados en un proceso productivo y cómo es la rentabilidad de la actividad (GERON et al., 2014).

Así, el objetivo de este trabajo fue realizar el análisis económico del costo de producción de leche, a través de la implementación de pastoreo rotacional, e intercambio de pasto nativo por pasto de Brachiaria brizantha $c v$. marandu, en una finca agrícola familiar.

RC: 99520

Disponible: https://www.nucleodoconhecimento.com.br/veterinaria-es/pastoreorotado 


\section{MATERIAL Y MÉTODOS}

El experimento se llevó a cabo en una propiedad lechera ubicada en la ciudad de

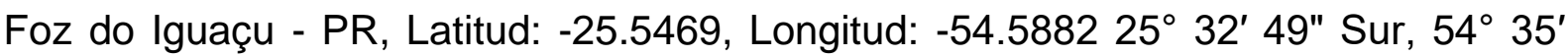
18" Oeste. Esta propiedad está compuesta por una superficie de 5.0 hectáreas, en esta zona se desarrolló un ganado lechero tradicional a la región, donde los animales tenían acceso a toda la zona de pastoreo, compuesta por pasto nativo de baja productividad, no había disponibilidad de sal mineral, solo sal común en el abrevadero y estando solo disponible una suplementación al momento del ordeño de $10 \mathrm{~kg}$ de animales lactantes y el acceso al agua era desde un arroyo. que pasa por la propiedad.

La propiedad cuenta con seis vacas lecheras, compuestas por cuatro vacas adultas, dos vacas lactantes y dos vacas secas, además de dos vaquillonas. Estos animales fueron manejados en toda el área de pasto durante todo el año y los animales lactantes recibieron $10 \mathrm{~kg}$ de alimento industrializado por día.

El trabajo comenzó con una visita a la propiedad para un diagnóstico. Las mediciones del área de pasto se realizaron utilizando GPS (Figura 1) y también recolección de suelo (Figura 2). Los resultados del análisis de suelos son (Tabla 1).

RC: 99520

Disponible: https://www.nucleodoconhecimento.com.br/veterinaria-es/pastoreorotado 
Figura 1 - Foto satelital del área experimental, con la demarcación del área de pastos:

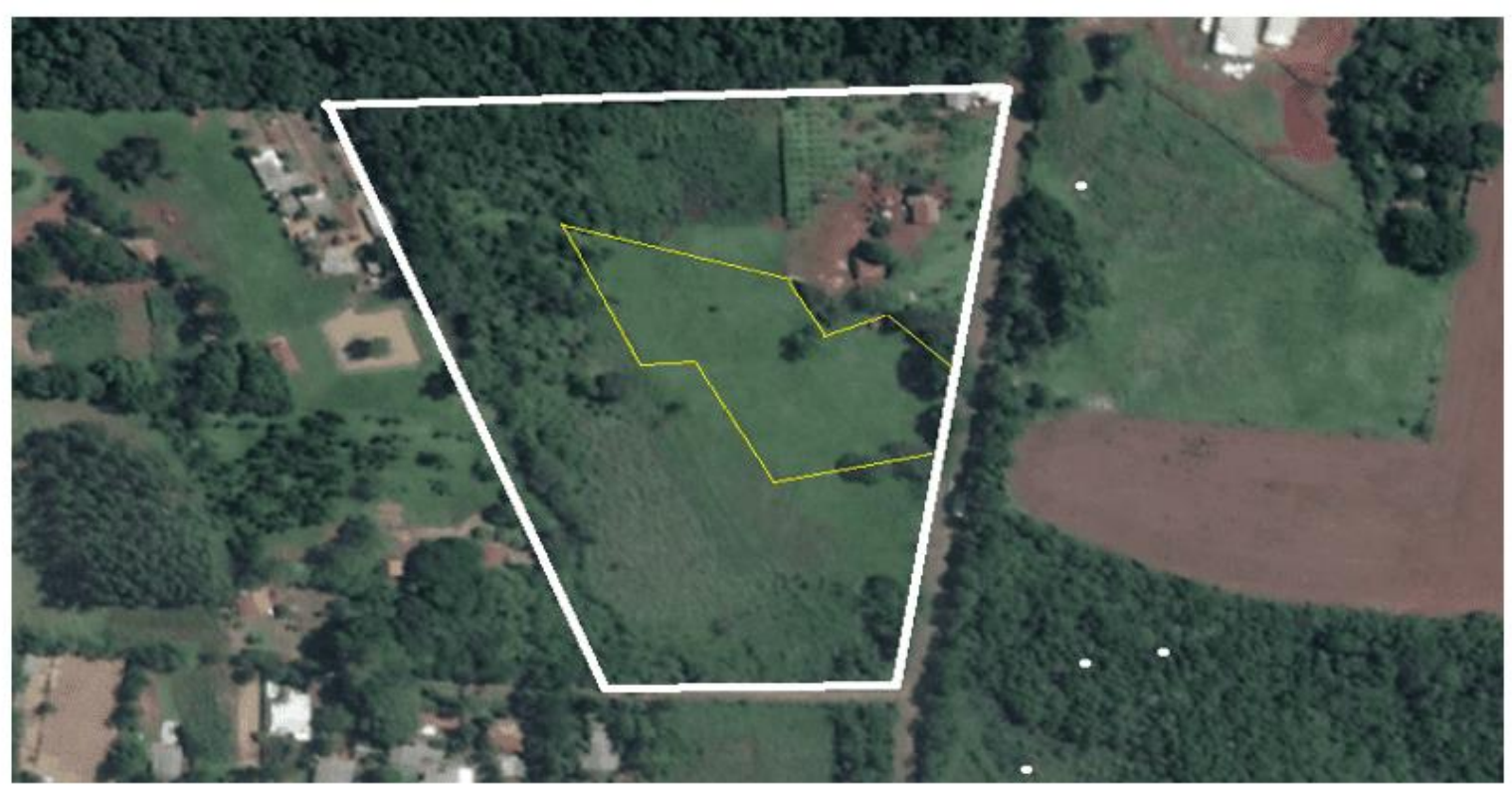

Fuente: Google Earth.

Figura 2 - Recolección de muestras de suelo:

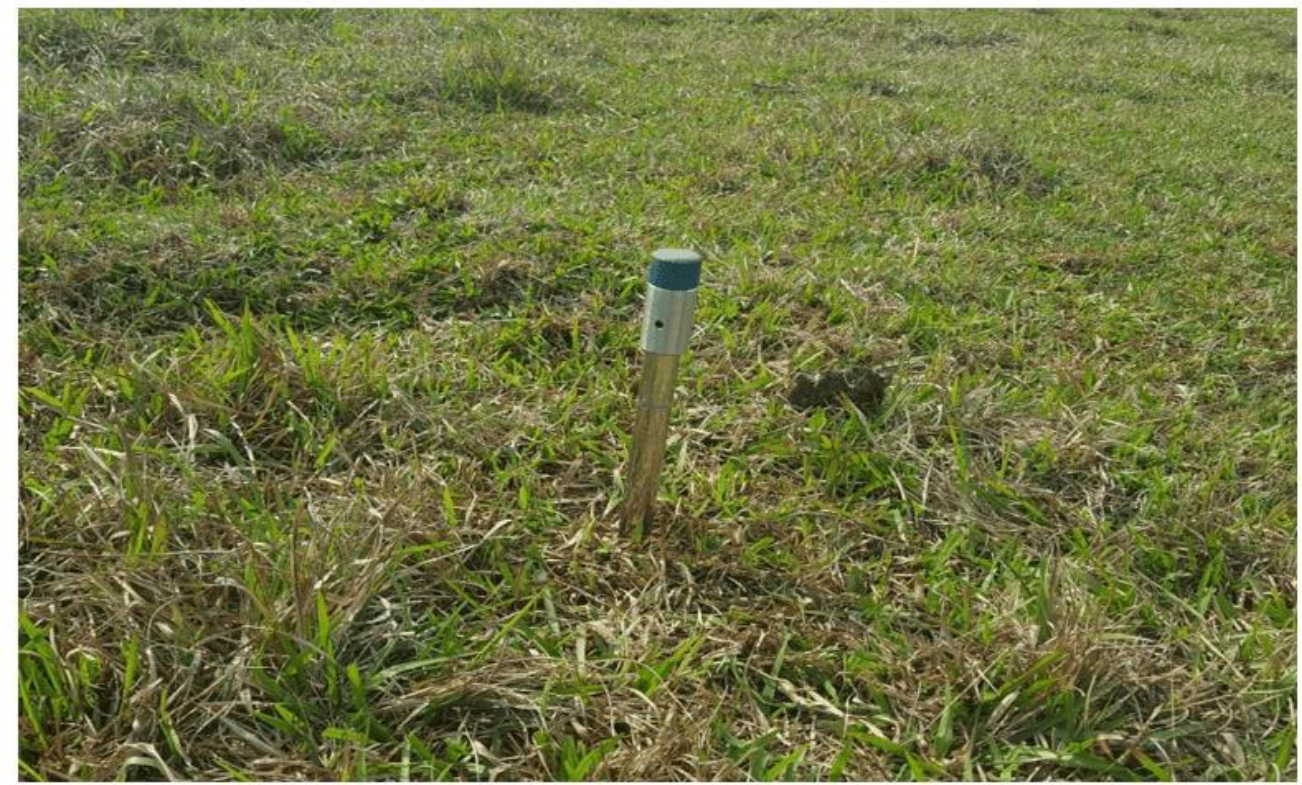

Fuente: Archivo Personal.

RC: 99520

Disponible: https://www.nucleodoconhecimento.com.br/veterinaria-es/pastoreorotado 
Tabla 1 - Resultados del análisis de suelos del área experimental:

\begin{tabular}{|c|c|c|c|c|c|c|c|c|c|c|c|c|c|c|}
\hline $\mathrm{pH}$ & & & $\mathrm{K}$ & $\mathrm{Ca}$ & $\mathrm{Mg}$ & $\mathrm{Al}$ & $\mathrm{H}+\mathrm{Al}$ & CTC & SB & V & $\mathrm{m}$ & $\mathrm{Ca}$ & $\mathrm{Mg}$ & $\mathrm{K}$ \\
\hline \multirow[t]{2}{*}{$\mathrm{CaCl} 2$} & M.O & $\mathrm{P}$ (res) & & & & & & & & & & & & \\
\hline & $\mathrm{g} / \mathrm{Kg}$ & $\mathrm{mg} / \mathrm{dm} 3$ & \multicolumn{5}{|c|}{$\mathrm{mmol} / \mathrm{dm} 3$} & $\%$ & & & \multicolumn{4}{|c|}{$\%$ СТС } \\
\hline 5,2 & 38 & 14 & 3 & 21 & 5 & 2,7 & 29 & 58 & 29 & 50 & 8 & 36 & 9 & 5 \\
\hline
\end{tabular}

Fuente: FZEA/USP.

Luego de recibir el análisis de suelo, se realizaron cálculos de corrección de suelo, donde el V\% fue $58 \%$. Para corregir el pH del suelo fueron necesarios $800 \mathrm{~kg}$ ha-1 de caliza dolomítica con $86 \%$ de PRNT. Para corregir el fósforo, fue necesario agregar $250 \mathrm{~kg} \mathrm{ha}^{-1}$ de fertilizante superfosfato simple. Para la fertilización de la producción se utilizaron $250 \mathrm{~kg} \mathrm{ha}^{-1}$ de urea agrícola (FRASSETO, 2015).

Para iniciar la preparación del suelo, se aplicó la aplicación de piedra caliza en toda el área experimental, y poco después, se utilizó una rejilla pesada, con una profundidad de $40 \mathrm{~cm}$, que sirvió para incorporar piedra caliza al suelo, romper compactaciones superficiales y dar condiciones al crecimiento de las nuevas especies forrajeras.

Para un dimensionamiento del área requerida para el pastoreo, las vacas se pesaron a través de una cinta de pesaje con una precisión del $95 \%$, totalizando $1.950 \mathrm{~kg} o$ 4,5 UA. Debido al hecho de que se utilizó ganado lechero, se determinó que los animales tendrían acceso a un nuevo piquete al final de un día de pastoreo. Por lo tanto, se utilizaron datos de (FRASSETO 2015), en los que un UA (450 kg de peso vivo) requiere al menos $50 \mathrm{~m}^{2}$ por día de área de pastoreo. Sabiendo que había 4,5 $\mathrm{UA}$, el área de cada piquete era de $225 \mathrm{~m}^{2}$. Se construyeron un total de 28 piquetes, utilizando 0,63 hectáreas. Durante la construcción de los piquetes, fue necesario agregar tres corredores, de $2 \mathrm{~m}$ de ancho.

RC: 99520

Disponible: https://www.nucleodoconhecimento.com.br/veterinaria-es/pastoreo$\underline{\text { rotado }}$ 
Para la división de los piquetes se utilizaron 50 mourões, 72 barras de refuerzo de $10 \mathrm{~mm}, 32$ barras de refuerzo de $12 \mathrm{~mm}, 500 \mathrm{~m}$ de alambre tri galvanizado para aproximadamente el perímetro total del área y $2500 \mathrm{~m}$ de alambre electroplástico para la división interna de los piquetes, 150 aislantes tipo marrón, 22 aisladores de gancho y 114 aislantes tipo barra de refuerzo. Se utilizó alambre de alambre con una altura de $80 \mathrm{~cm}$ en relación con la tierra y cada $10 \mathrm{~m}$ se implantó una barra de refuerzo de 1,5 m con aislante para mantener la altura en relación con la tierra, también se utilizó alambre electroplástico para dividir los piquetes y reducir los costos de formación. Para electrificar el cable, se utilizó un electrificador Sentinel modelo 30.000, con un mínimo de 5000 voltios.

Figura 3 - Croquis de la división de los piquetes:

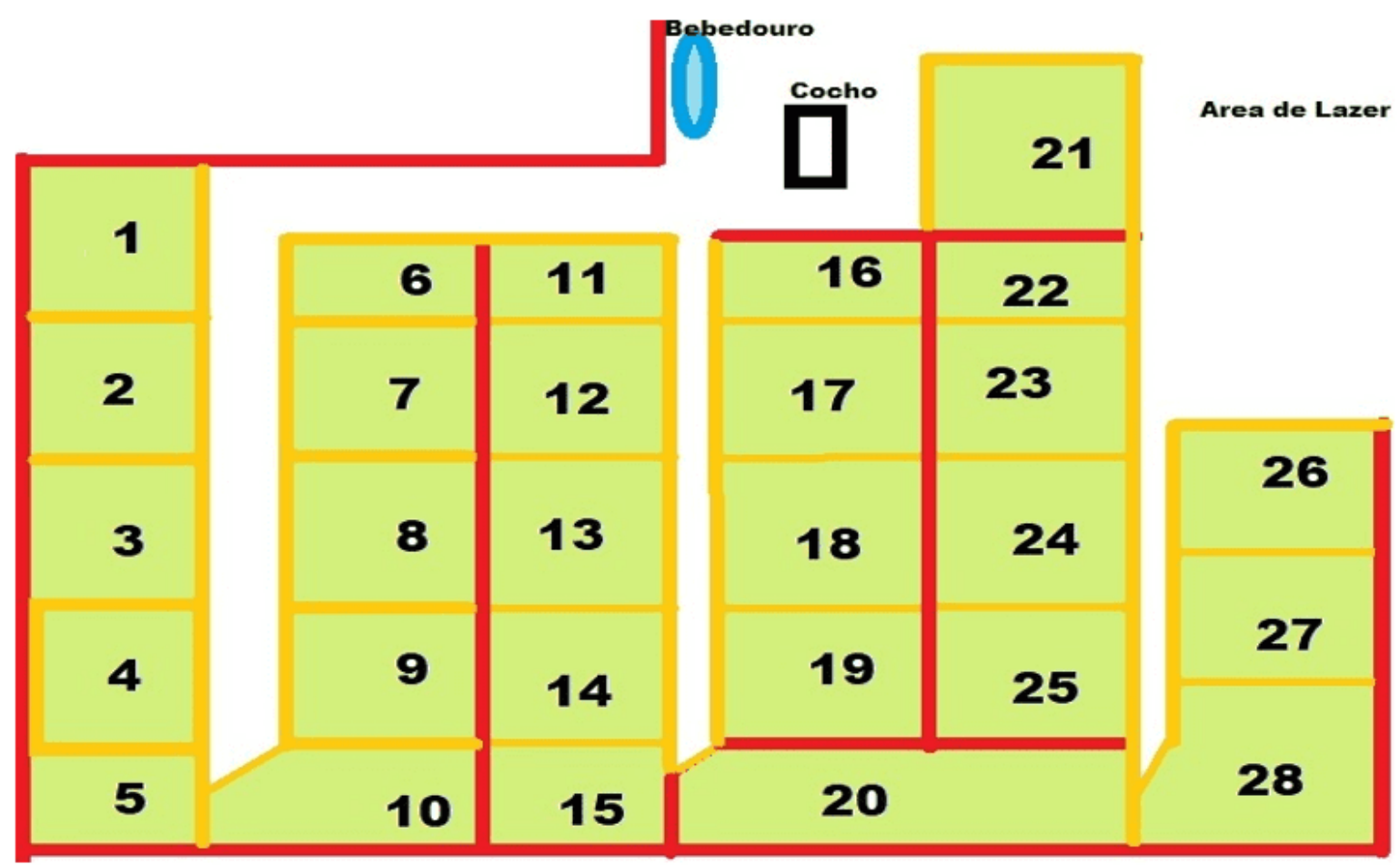

Fuente: Archivo personal.

El forraje elegido fue Brachiaria Brizantha cv. marandu, el manejo de este forraje comienza cuando la planta alcanza los $30 \mathrm{~cm}$ de entrada y los $15 \mathrm{~cm}$ de salida de

RC: 99520

Disponible: https://www.nucleodoconhecimento.com.br/veterinaria-es/pastoreorotado 
Andrade (2008); Fonseca (2010). La siembra de forraje y la fertilización de siembra comenzaron el 15/11/2019, con el método de siembra e incorporación de semillas y fertilizante de fosfato supersimple. Las semillas fueron incrustadas, y se utilizó 15 $\mathrm{kg} \mathrm{ha}^{-1}$.

La primera fertilización de producción se realizó 60 días después de la siembra, aplicando $125 \mathrm{~kg} \mathrm{ha}^{-1}$ de urea agrícola con $45 \%$ de nitrógeno. El primer pastoreo comenzó el 03/02/2020, 77 días después de la siembra. La segunda fertilización de producción aplicándose $125 \mathrm{Kg} \mathrm{ha}^{-1}$ de urea agrícola se realizó en el primer ciclo de pastoreo siendo 28 días después del inicio del primer pastoreo.

Para el resto de los animales se agregó un bebedero de agua con aproximadamente $100 \mathrm{~L}$ de agua, adición de sal mineral apta para ganado lechero y los propios árboles de la propiedad sirvieron de sombreado.

Para realizar el análisis económico y la evaluación del costo de producción de leche, se identificaron los agentes involucrados, así como los valores que representan los efectos de la implementación del proyecto sobre estos agentes, considerando también el cierre de un año.

Para evaluar el costo de implementación del proyecto, consideramos los costos de invertir en la preparación del suelo y la siembra del pasto, así como las inversiones en infraestructura y equipo en la formación de piquetes.

Para evaluar el costo de producción, se utilizó la estructura del costo operativo de producción, propuesta por Matsunaga (1976). En el cual, el costo operativo efectivo (COE), corresponde al gasto de dinero por parte del productor, con compra de alimento, gastos con el mantenimiento de pastos y medicamentos, durante un año.

Para el costo total de producción (CTP), se agregó la depreciación a (COE). La depreciación anual del pasto se calculó por la suma de los suministros necesarios

RC: 99520

Disponible: https://www.nucleodoconhecimento.com.br/veterinaria-es/pastoreorotado 
para la construcción de la cerca eléctrica dividida en un período de diez años. Depreciación del 10\% anual (CANZIANI et al., 2000).

Para calcular los ingresos, se consideraron los ingresos netos, que es la cantidad ganada en la entrega de leche por año menos los costos totales de producción (MARTIN, 1997).

\section{RESULTADOS Y DISCUSIÓN}

Dentro de la producción agrícola, es necesario tener una planificación por parte del productor, para que haya crecimiento y desarrollo de la actividad. Por lo tanto, el primer paso en el estudio de la viabilidad de un proyecto es estudiar los gastos para su implementación. Los costos para la implementación de este proyecto, desde la preparación del suelo para la siembra del pasto, hasta la formación de los piquetes fueron de $\mathrm{R} \$ 3.023,70$.

Antes de realizar la implantación de hierba $B$. brizantha cv. marandu, era necesario preparar el suelo. Las inversiones relacionadas con la labranza del suelo se describen en (Tabla 2).

Tabla 2 - Costos de inversión en preparación y siembra del suelo:

\begin{tabular}{|c|c|c|c|}
\hline Descripción & Unidad & Cantidad & Valor $(\mathrm{R} \$)$ \\
\hline Análisis del suelo & Uni & 1 & 33,00 \\
\hline $\begin{array}{l}\text { Piedra caliza dolomita } \text { (PRNT } \\
86 \%)\end{array}$ & $\mathrm{Kg}$ & 800 & 156,00 \\
\hline Superfosfato (19.00.00) & $\mathrm{Kg}$ & 250 & 287,46 \\
\hline Tiempo de aración del tractor & $\mathrm{H}$ & 2 & 400,00 \\
\hline Semilla incrustada & $\mathrm{Kg}$ & 15 & 287,01 \\
\hline Urea agrícola & $\mathrm{Kg}$ & 250 & 400,00 \\
\hline Herbicida Glifosato & $\mathrm{Ml}$ & 500 & 35,00 \\
\hline
\end{tabular}

RC: 99520

Disponible: https://www.nucleodoconhecimento.com.br/veterinaria-es/pastoreorotado 


\begin{tabular}{|l|l|l|l|}
\hline Aceite 2T & Ml & 250 & 20,00 \\
\hline Combustible - Gasolina & L & 5 & 20,00 \\
\hline Total & & & $\mathbf{1 . 6 3 8 , 4 7}$ \\
\hline
\end{tabular}

Fuente: Datos del proyecto.

Los costos de labranza del suelo y la siembra representaron el $54,20 \%$ de los costos totales para la implantación de pastos. Las prácticas correctivas y la fertilización del suelo son solo una parte de los requisitos necesarios para el éxito del sistema de producción de pastos (PEREIRA et al., 2018).

Es importante que el productor invierta y realice una buena preparación del suelo y una correcta implementación del pasto, buscando siempre el equilibrio en el sistema suelo-planta-animal (PEREIRA et al., 2018). Estos manejos influirán en el aumento de la producción animal, así como en la reducción de los gastos de alimentación animal.

En la búsqueda de un correcto manejo del pasto, se subdividió en piquetes. Las inversiones en infraestructura y equipamiento en la formación de piquetes están representadas en (Tabla 3).

Tabla 3 - Tabla de costos para la formación de piquetes, con la cerca eléctrica:

\begin{tabular}{|l|l|l|l|}
\hline Descripción & Unidad & Cantidad & Valor (R\$) \\
\hline Alambre Eletrix & $\mathrm{m}$ & 500 & 184,00 \\
\hline $\begin{array}{l}\text { Alambre electroplástico 500 } \\
\mathrm{m}\end{array}$ & $\mathrm{m}$ & 5 & 104,82 \\
\hline $\begin{array}{l}\text { Aislante marrón } \\
\begin{array}{l}\text { Aislante de barras de } \\
\text { refuerzo }\end{array}\end{array}$ & Uni & 150 & 176,98 \\
\hline \begin{tabular}{l} 
Aislante tipo gancho corto \\
\hline
\end{tabular} & Uni & 20 & 24,00 \\
\hline
\end{tabular}

RC: 99520

Disponible: https://www.nucleodoconhecimento.com.br/veterinaria-es/pastoreorotado 


\begin{tabular}{|l|l|l|l|}
\hline Aislante tipo gancho largo & Uno & 2 & 4,60 \\
\hline $\begin{array}{l}\text { Interruptor de conmutación } \\
\text { Barras de refuerzo (3/8") }\end{array}$ & Uno & 3 & 36,00 \\
\hline $\mathrm{m}$ & Uno & 72 & 284,30 \\
\hline $\begin{array}{l}\text { Barras de refuerzo (1/2") } \\
\text { m }\end{array}$ & Uno & 2 & 186,18 \\
\hline Tubo de PVC 20 mm 6 m & Uno & 1 & 12,80 \\
\hline Voltímetro digital & Uno & 1 & 139,90 \\
\hline Electrificador & Uno & 1 & 100,00 \\
\hline Total & & & $\mathbf{1 . 3 8 5 , 2 3}$ \\
\hline
\end{tabular}

Fuente: Datos del proyecto.

Los costos de infraestructura y equipamiento para la formación de piquetes representan el $45,80 \%$ de los costos totales de la implementación del pasto. El manejo racional del forraje ha demostrado ampliamente los beneficios de dividir los pastos, donde existe una mayor uniformidad del pastoreo, mayor utilización de forrajes, mayor tasa de siembra y mayor longevidad de las malezas que forman el grupo (FUKUMOTO, 2010), que es el caso de B. brizantha $c v$. marandu. El uso de la cerca eléctrica, en sus diferentes formas en piquetes, es una herramienta necesaria para implementar a bajo costo las subdivisiones que requiere el pastoreo rotacional (EMBRAPA, 1999).

Los activos que componen la empresa están sujetos a constantes devaluaciones, principalmente debido al desgaste y al envejecimiento. (Tabla 4) tiene los datos de depreciación de la cerca eléctrica.

Cuadro 4 - Depreciación de materiales y equipo:

\begin{tabular}{|lll|l|l|l|}
\hline Descripción & & Monto total R\$ & Vida útil (años) & Valor anual \\
\hline Construcción de cercas & $1.385,25$ & $\mathbf{1 0}$ & 138,52 \\
\hline
\end{tabular}

RC: 99520

Disponible: https://www.nucleodoconhecimento.com.br/veterinaria-es/pastoreorotado 
eléctricas

Fuente: Datos del proyecto.

Cuadro 5 - Pago de la inversión:

\begin{tabular}{|c|c|c|c|}
\hline Descripción & Monto total $\mathrm{R} \$$ & Tiempo de pago (años) & Valor anual \\
\hline $\begin{array}{ll}\text { Costo } & \text { de } \\
\text { implementación } & \end{array}$ & $3,023.70$ & 10 & 302.37 \\
\hline
\end{tabular}

Fuente: Datos del proyecto.

Para tener un mayor control anual sobre la gestión empresarial, las inversiones para el despliegue se dividieron en 10 años. 4,20\% de los ingresos anuales por la venta de leche, descritos en (Tabla 5).

Para calcular la ingesta monetaria, en forma de dinero en un año, se consideró la cantidad pagada al productor por litro de leche, vendida diariamente y directamente del productor de la forma durante un año. Para calcular la cantidad de leche anual se consideró un ordeño diario por la mañana con un total de 10 litros/día (Tabla 6).

Cuadro 6 - Ingresos procedentes de la venta de leche anual:

\begin{tabular}{|l|l|}
\hline Variables & Valor total \\
\hline Precio por litro $(\mathrm{R} \$)$ & 2,00 \\
\hline $\begin{array}{l}\text { Litros totales de leche producidos } \\
(\mathrm{L})\end{array}$ & $3.600,00$ \\
\hline Ingresos finales $(\mathrm{R} \$)$ & $\mathbf{7 . 2 0 0 , 0 0}$ \\
\hline
\end{tabular}

Fuente: Datos del proyecto.

RC: 99520

Disponible: https://www.nucleodoconhecimento.com.br/veterinaria-es/pastoreo$\underline{\text { rotado }}$ 
Cada año el productor tendrá que hacer un gasto de dinero, que se considera un Costo Operativo Efectivo (COE). Se tuvieron en cuenta los costos de alimentación, los gastos con el mantenimiento de los pastos y los medicamentos (Tabla 7).

Tabla 7 - Costo Operativo Efectivo - COE, por año:

\begin{tabular}{|l|l|l|l|l|}
\hline Descripción & Unidad & Quant & Valor unitario R\$ & Monto total R\$ \\
\hline Ración & $\mathrm{Kg}$ & 720 & 1,32 & 950,04 \\
\hline Urea agrícola & $\mathrm{Kg}$ & 250 & 1,60 & 400,00 \\
\hline Energía eléctrica & $\mathrm{R} \$$ & 12 & 0,60 & 216,00 \\
\hline Vacuna contra la brucelosis & Dosis & 6 & 1,62 & 9,72 \\
\hline Vacuna contra la rabia & Dosis & 6 & 1,48 & 8,88 \\
\hline Dectomax 50 mL & Uni. & 1 & 21,90 & 21,90 \\
\hline $\begin{array}{l}\text { Vacuna contra la fiebre } \\
\text { aftosa }\end{array}$ & Dosis & 6 & 1,50 & 9,00 \\
\hline Total & & & & \\
\hline
\end{tabular}

Fuente: Datos del proyecto.

Se observa en este trabajo que el mayor gasto en producción es en piensos. Según (MARTINEZ, 2009), el gasto en nutrición representa en promedio el $67 \%$ de los costos de producción de leche en Brasil. Por lo tanto, se buscan estrategias para reducir el uso de dietas y otros suplementos. Mejorar los pastos y gestionarlo es una alternativa que ayuda a que la producción de leche sea económicamente viable. Por esta razón, el pastoreo rotacional ha sido cada vez más indicado (MARION et al., 2010).

Rosestolato (2015), al analizar la viabilidad económica de la producción lechera de dos propiedades distintas respecto al sistema de producción tecnológica, observó que el (COE) en la propiedad en la que las vacas se mantenían en un sistema de pastoreo continuo y se complementaba con alimento concentrado y sal mineral era

RC: 99520

Disponible: https://www.nucleodoconhecimento.com.br/veterinaria-es/pastoreorotado 
mayor que el mantenido en un sistema de pastoreo rotacional y complementado con caña de azúcar picada, pienso concentrado y sal mineral.

En la propiedad evaluada en este estudio, se dieron $10 \mathrm{~kg}$ de alimento industrializado por animal lactante y después de la implementación del proyecto hubo una disminución de $9 \mathrm{~kg}$ comenzando a ofrecer solo $1 \mathrm{~kg}$ de alimento a los animales, disminuyendo así el (COE), como observó Rosestolato (2015). Sin embargo, la suplementación a los animales incluso con el cambio en el sistema de pastoreo es importante, ya que ayuda a suministrar en los animales los nutrientes que faltan en la composición del forraje. El costo del alimento representó el 44,70\% de todos los costos de producción.

Considerando los valores de depreciación y costo efectivo de operación (COE), el costo total de producción se obtuvo en un año (Tabla 8).

Tabla 8 - Costo total de producción - CTP (Base anual):

\begin{tabular}{|l|l|}
\hline Descripción & Monto total R\$ \\
\hline $\begin{array}{l}\text { Depreciación } \\
\text { Costos de COE }\end{array}$ & 138,52 \\
\hline $\begin{array}{l}\text { Pago de la } \\
\text { inversión }\end{array}$ & 302.37 \\
\hline Total & $\mathbf{2 . 0 5 6 , 3 9}$ \\
\hline
\end{tabular}

Fuente: Datos del proyecto.

En los países con precios bajos de la leche, los productores pueden reducir el costo de producción al aumentar la participación de los pastos en la dieta de las vacas lecheras. En este estudio, el costo total de producción representó el $28,55 \%$ de los ingresos anuales.

RC: 99520

Disponible: https://www.nucleodoconhecimento.com.br/veterinaria-es/pastoreorotado 
Para calcular los ingresos, se consideraron los ingresos netos, que es el valor obtenido en la entrega de leche por año menos los costos totales de producción (Tabla 9).

Cuadro 9 - Ingresos (base anual):

\begin{tabular}{|l|l|}
\hline Descripción & Monto total R\$ \\
\hline Ingresos & $7.200,00$ \\
\hline $\begin{array}{l}\text { Costo total de producción(base anual } \\
\text { CTP) }\end{array}$ & $\mathbf{2 . 0 5 6 , 3 9}$ \\
\hline Equilibrar & $\mathbf{5 . 1 4 3 , 6 1}$ \\
\hline
\end{tabular}

Fuente: Datos del proyecto.

Según Oliveira (2007), el conocimiento de las implicaciones del costo operativo efectivo (COE), en el corto plazo, es fundamental en la gestión del negocio, y el margen bruto debe ser positivo, si es negativo, se recomienda la interrupción de la producción.

El análisis financiero de la ejecución de este proyecto resultó factible (Cuadro 9). A la vista de los análisis realizados a partir de los datos obtenidos, se observó que la inversión en la técnica de pastoreo rotacional en ganado lechero presenta un retorno económicamente viable sobre el capital invertido y que la misma influencia positiva en la producción hace que esta actividad sea más competitiva en el sector. Así, esta técnica permite la expresión potencial para la producción de leche y el aumento de la rentabilidad de los productores, lo que rentabilizaría la actividad láctea, incluso en épocas de caída del precio de la leche.

\section{CONSIDERACIONES FINALES}

De acuerdo con los resultados obtenidos en los análisis económicos, se concluye que la implementación del pastoreo rotacional y el intercambio de pastos nativos por

RC: 99520

Disponible: https://www.nucleodoconhecimento.com.br/veterinaria-es/pastoreorotado 
Brachiaria brizantha cv. marandu, es una gestión viable, ya que los ingresos generados por la venta de leche fueron suficientes para cubrir los costos totales de producción.

Este sistema de producción semi-intensivo es recomendado a la realidad local, porque es una alternativa de innovación tecnológica, capaz de reducir gastos con la compra de piensos, y proporcionar un buen desempeño animal, a partir del cambio de manejo del pastoreo e inserción de pasto de mejor valor nutricional y mayor producción en masa.

\section{REFERENCIAS}

ANDRADE, Carlos Mauricio Soares de. Pastejo Rotacionado: Tecnologia para Aumentar a Produtividade de Leite e a Longevidade das Pastagens. EMBRAPA - Acre, dez. 2008.

ANUÁRIO LEITE 2019. Sua excelência, o consumidor: novos produtos e novas estratégias da cadeia do leite para ganhar competitividade e conquistar os clientes finais. Brasília, DF: Texto Comunicação Corporativa, 2019. Disponível em: $<$ https://www.infoteca.cnptia.embrapa.br/infoteca/handle/doc/1109959>. Acesso em: 25 mar. 2020.

DEMSKI, Joana Baptista. Desempenho e comportamento de vacas lactantes em pastagens de cultivares de braquiárias. Dissertação apresentada ao Programa de Pós-graduação do Instituto de Zootecnia, APTA/SAA. Nova Odessa - SP, 2013. $80 \mathrm{p}$.

FONSECA, Delermando Miranda da; MARTUSCELLO, Janaina Azevedo. Plantas forrageiras. Viçosa, 537p. 2010.

FUKUMOTO, Nelson Massaru; DAMASCENO, Júlio Cesar; DERESZ, Fermino, MARTINS, Carlos Eugênio; CÓSER, Antônio Carlos; SANTOS, Geraldo Tadeu dos.

RC: 99520

Disponible: https://www.nucleodoconhecimento.com.br/veterinaria-es/pastoreorotado 
Produção e composição do leite, consumo de matéria seca e taxa de lotação em pastagens de gramíneas tropicais manejadas sob lotação rotacionada. Revista Brasileira de Zootecnia, v. 39, n.7, p. 1548-1557, 2010.

GERON, Luiz Juliano Valério; MEXIA, Alexandre Agostinho; GARCIA Jocilaine; SILVA Marciano Moreira da; ZEOULA, Lúcia Maria. Suplementação concentrada para cordeiros terminados a pasto sobre custo de produção no período da seca. Semina: Ciências Agrárias, Londrina, v. 33, p. 797-808, 2012.

GERON, Luiz Juliano Valério; MOURA, Daiane Caroline; RODRIGUES, Deivison Novaes; PAULA, Edson Júnior Heitor; TRAUTMANN-MACHADO, Raquel Joana; GARCIA, Jocilaine; SCHUMANN, Alline Mariá; SILVA; Dilma Alves. Viabilidade econômica de tourinhos terminados em confinamento alimentados com diferentes teores de caroço de algodão em dietas elaboradas com coprodutos agroindustriais. Semina: Ciências Agrárias, Londrina, v.35, p. 2673-2684, 2014.

GONÇALVES, Carlos Alberto; CAMARÃO, Ari Pinheiro; DUTRA, Saturnino; AZEVEDO, Guilherme Pantoja Calandrini de; MENDONÇA, Denise Castro; SOBRINHO, Carlos de Melo Junior. Produção de leite em pastejo rotacionado intensivo de "Brachiaria brizantha" cv. Marandu sob dois níveis de suplementação concentrado. In: Reunião anual da sociedade brasileira de zootecnia, 40., 2003, Santa Maria. Anais... Santa Maria: Sociedade Brasileira de Zootecnia, 2003

IBGE -Instituto Brasileiro de Geografia e Estatística. [2018]. Pesquisa da pecuária nacional disponível em: <https://sidra.ibge.gov.br/Tabela/74>. Acessado em: 25 mar. 2020.

IPARDES: Instituto Paranaense de Desenvolvimento Econômico e Social. Caracterização sócio econômica da atividade leiteira no Paraná: Sumário executivo. Curitiba: IPARDES, 2009. 29 p.

RC: 99520

Disponible: https://www.nucleodoconhecimento.com.br/veterinaria-es/pastoreorotado 
JANK, Liana; ANDRADE, Carlos Mauricio Soares de; BARBOSA, Rodrigo Amorim; MACEDO, Manuel Claudio Motta; VALERIO, Jose Raul; VERZIGNASSI, Jaqueline Rosemeire; ZIMMER, Ademir Hugo; FERNANDES, Celso Dornelas; SANTOS, Mateus Figueiredo; RESENDE, Rosangela Maria Simeão. O capim-BRS Quênia (Panicum maximum Jacq.) diversificación e intensificación de los pastos. Disponible en: <https: ainfo.cnptia.embrapa.br/digital/bitstream/item/165106/1/capimbrs-quenia-panicum-maximum-jacq.pdf="'>.</https:> Acceso: 25 Mar. 2020.

MARION, Jose Carlos; SEGATTI, Sonia. Contabilidade da Pecuária. 9 ed. São Paulo: Atlas, 2010.

MARTIN, Nelson Batista; SERRA, Renata; OLIVEIRA, Marli Dias Mascarenhas; ÂNGELO, José Alberto; OKAWA, Hiroshige. Sistema "CUSTAGRI”: sistema integrado de custos agropecuários. São Paulo: IEA/SAA, 1997. p. 1-75.

MARTINEZ, Junior Cesar. Produção de leite a pasto: manejo do pastejo é fundamental para manter a produção. 2009. Disponível em: <http://www.milkpoint.com.br/radartecnico/nutricao/producao-deleite-a-pasto-manejodo-pastejo-e-fundamental-para-manter-aproducao-51710n.aspx>. Acesso em: 27 mai. 2020.

MATSUNAGA, Minoru; BEMELMANS, Paul Frans; TOLEDO, Paulo Edgard Nascimento de; DULLEY, Richard Domingues; HIROSHIGE, Okawa; PEDROSO, Iby Arvatti. Metodologia de custo utilizada pelo IEA. Agricultura em São Paulo, v. 23, n. 1, p.123-39, 1976.

OLIVEIRA, Jefferson Soares de. Análise de eficiência e alocação de recursos na produção leiteira do estado do Rio de Janeiro. In: congresso da sociedade brasileira de economia, administração e sociologia rural, 45., 2007. Londrina. Conhecimentos para a agricultura do futuro: Anais... Brasília, DF: SOBER; Londrina: IAPAR: Universidade Estadual de Londrina, 2007.

RC: 99520

Disponible: https://www.nucleodoconhecimento.com.br/veterinaria-es/pastoreorotado 
PEREIRA, Lilian Elgalise Techio. et al. Recomendações para correção e adubação de pastagens tropicais. Pirassununga: Faculdade de Zootecnia e Engenharia de Alimentos da USP, 2018. 56p.

ROSESTOLATO, Lucas Luiz Rocha. et al. Viabilidade econômica comparativa de sistemas tecnológicos da pecuária leiteira no município de llha Solteira/SP. Congresso de extensão universitária da UNESP, 8., p. 1-6, 2015. Disponível em: <http://hdl.handle.net/11449/142491>. Acesso em: 28 mai. de 2020.

SEAB - Secretaria de Estado da Agricultura e do Abastecimento. Bovinocultura de leite: leite - $\quad$ produção $2016 / 17 . \quad$ Disponível em:<http://www.agricultura.pr.gov.br/arquivos/File/deral/Prognosticos/2018/leite_201 7_18.pdf>. Acesso em: 25 mar. de 2020.

Publicado: Julio de 2021.

Aprobado: Septiembre de 2021.

RC: 99520

Disponible: https://www.nucleodoconhecimento.com.br/veterinaria-es/pastoreorotado 